\title{
EFEITO DE INSETICIDAS EM DIFERENTES FASES DE DESENVOLVIMENTO DE Grapholita molesta (BUSCK, 1916) (LEPIDOPTERA: TORTRICIDAE) E ESTRUTURAS VEGETAIS DA MACIEIRA E DO PESSEGUEIRO' ${ }^{1}$
}

\author{
CINDY CORRÊA CHAVES 2 , CLÉBER ANTONIO BARONIO ${ }^{3}$, \\ MARCOS BOTTON ${ }^{4}$, MAURO SILVEIRA GARCIA $^{5}$
}

RESUMO - A mariposa-oriental Grapholita molesta é uma das principais pragas da macieira e do pessegueiro. Neste trabalho, foi avaliada a mortalidade causada por inseticidas quando aplicados sobre diferentes fases de desenvolvimento de G. molesta e sobre ponteiros e frutos de macieira e pessegueiro. Os inseticidas

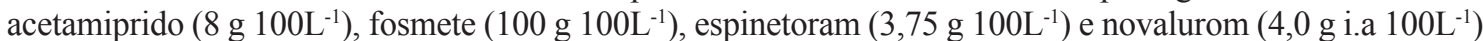
reduziram a eclosão em níveis superiores a $80 \%$, independentemente de a aplicação ser realizada em pré ou pós-oviposição. O etofenproxi $\left(15 \mathrm{~g} 100 \mathrm{~L}^{-1}\right)$ foi mais eficaz quando aplicado em pós-oviposição. Em

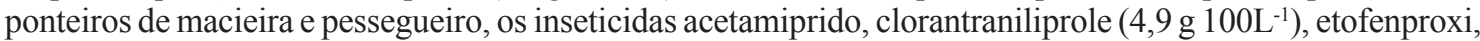
fosmete, novalurom e espinetoram causaram mortalidade de lagartas acima de $90 \%$. Em frutos de macieira, os inseticidas acetamiprido, clorantraniliprole, etofenproxi, fosmete e espinetoram causaram mortalidade de lagartas de 100; 79; 76; 97 e 100\%, respectivamente, enquanto em frutos de pessegueiro apresentaram controle superior a $85 \%$. O inseticida novalurom aplicado em frutos causou menor mortalidade das lagartas quando comparado à aplicação em ponteiros. Em adultos, apenas os inseticidas etofenproxi e fosmete foram tóxicos tanto a fêmeas (59 e 39\%) quanto a machos (79 e 80\%), enquanto o espinetoram mostrou efeito apenas em machos (78\%). Conclui-se que os inseticidas etofenproxi, fosmete e espinetoram foram eficientes no controle de G. molesta em todas as fases de desenvolvimento.

Termos para indexação: Mariposa-oriental, Acetamiprido, Clorantraniliprole, Espinetoram, Etofenproxi, Novalurom.

\section{EFFECT OF INSECTICIDES IN DIFFERENT STAGES OF DEVELOPMENT OF Grapholita molesta (BUSCK, 1916) (LEPIDOPTERA: TORTRICIDAE) AND VEGETATIVE STRUCTURES OF APPLE AND PEACH}

\begin{abstract}
The oriental fruit moth Grapholita molesta is one of the main pests of apple and peach trees. In this study, the mortality caused by insecticides when applied on different stages of development of G. molesta

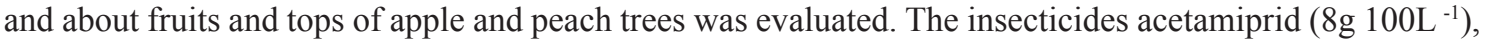

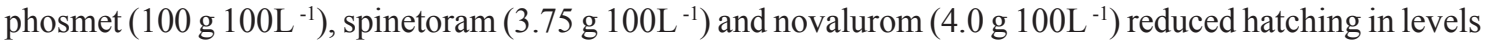
above $80 \%$, regardless of whether the application is performed before or after oviposition. The etofenprox

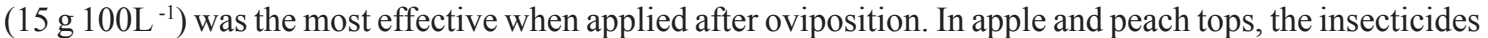

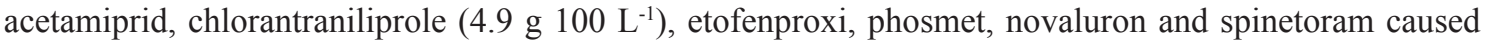
mortality of larvae over $90 \%$. In apple fruits, the insecticides acetamiprid, chlorantraniliprole, etofenprox, phosmet, and spinetoram, caused 100, 79, 76, 97 and 100\% mortality, respectively, whereas in peach fruits this control was over $85 \%$. The insecticide novaluron caused lower mortality of larvaes when applied on fruits, compared to the application on tops. In adults, only the insecticides etofenprox and phosmet were toxic both to females $(59 \%$ and $39 \%$ ) and males ( $79 \%$ and $80 \%$ ), while spinetoram was effective only in males $(78 \%)$. It is concluded that insecticides etofenprox, phosmet and spinetoram were efficient in the control of G. molesta in all stages of development.

Index terms: Oriental fruit moth, Acetamiprid, Chlorantraniliprole, Spinetoram, Etofenprox, Novaluron.

\footnotetext{
'(Trabalho 390-13). Recebido em:15-10-2013. Aceito para publicação em: 12-06-2014.

${ }^{2}$ Eng. Agra ${ }^{a}$ Mestranda do Depto. de Fitossanidade/FAEM/UFPel, Caixa Postal 354, CEP: 96010-900, Pelotas. E-mail: cindycchaves@ yahoo.com.br

${ }^{3}$ Eng. Agr ${ }^{\circ}$, Doutorando do Depto. de Fitossanidade/FAEM/UFPel, Caixa Postal 354, CEP: 96010-900, Pelotas. E-mail: cleber. baronio@hotmail.com

${ }^{4}$ Eng. Agr ${ }^{\circ}$, Dr. Pesquisador Embrapa Uva e Vinho, Caixa Postal: 130, CEP: 95700-000, Bento Gonçalves. E-mail: marcos.botton@ embrapa.br

${ }^{5}$ Eng. Agr ${ }^{0}$, Dr. Professor do Depto. de Fitossanidade/FAEM/UFPel, Caixa Postal 354, CEP: 96010-900, Pelotas. E-mail: garciasmauro@yahoo.com.br
}

Rev. Bras. Frutic., Jaboticabal - SP, v. 36, n. 4, p. 842-852, Dezembro 2014 


\section{INTRODUÇÃO}

A mariposa-oriental Grapholita molesta (Busck, 1916) (Lepidoptera: Tortricidae) é uma das principais pragas da macieira (Malus domestica Borkhausen) e do pessegueiro (Prunus persica (L.) Batsch) no Brasil (NORA; HICKEL, 2006; BOTTON et al.,2011). Os danos são causados pelas lagartas que se alimentam das brotações novas (ponteiros) e frutos (SALLES, 2001; MONTEIRO; HICKEL, 2004).

O controle da $G$. molesta é realizado principalmente com inseticidas fosforados que apresentam restrições em relação à alta toxicidade $\mathrm{e}$ potencial de deixar resíduos nos frutos (AGROFIT, 2013; MAPA, 2013). Além disso, a resistência de G. molesta a inseticidas organofosforados já foi registrada em alguns países (PREE et al., 1998; KANGA et al., 1999; SHEARER; USMANI, 2001; USMANI; SHEARER, 2001), inclusive no Brasil (SIEGWART et al., 2011). Por essas razões, o uso deste grupo químico está sendo revisto, e alguns estão sendo retirados do mercado (ANVISA, 2010), sendo substituídos por inseticidas de menor toxicidade e com menor impacto ao ambiente e à saúde humana.

Diferentes grupos químicos de inseticidas foram recentemente disponibilizados para o manejo de lagartas nas culturas da macieira e do pessegueiro com destaque para o neonicotinoide (acetamiprido), espinosina (espinetoram), antranilamida (clorantraniliprole), benzoilureia (novalurom), éter piridiloxipropílico (piriproxifem) e éter difenílico (etofenproxi) (AGNELLO et al., 2009; OMOTO et al., 2012; AGROFIT, 2013).

A maioria dos novos grupos químicos tem como alvo biológico a fase de lagarta que causa prejuízos aos cultivos (ARIOLI et al., 2004; SIQUEIRA; GRUTZMACHER, 2005; GUERRA et al., 2007; NUNES; MARONDI, 2007), embora os mesmos apresentem efeitos secundários sobre as outras fases de desenvolvimento da espécie (ASSAL et al., 1983; YOKOYAMA; MILLER, 1991; SPARKS et al., 2001; BASSI et al., 2009; MAGALHÃES; WALGENBACH, 2011).

A transição bem-sucedida do uso de organofosforados para os novos grupos químicos no manejo da G. molesta depende do conhecimento da ação dos inseticidas sobre as diferentes fases de desenvolvimento dos insetos, permitindo definir a melhor época de aplicação nos pomares. O objetivo deste trabalho foi avaliar o efeito de inseticidas sobre as diferentes fases de desenvolvimento de G. molesta e estruturas vegetais da macieira e do pessegueiro.

\section{MATERIAL E MÉTODOS}

Os experimentos foram conduzidos no Laboratório de Entomologia da Embrapa Uva e Vinho, localizado em Bento Gonçalves-RS, à temperatura de $25 \pm 1^{\circ} \mathrm{C}, \mathrm{UR}$ de $70 \pm 10 \%$ e fotofase de 16 horas. A população original dos insetos utilizados nos experimentos foi coletada em pomar de macieira localizado em Vacaria-RS (Latitude $28^{\circ} 26^{\prime} \mathrm{S}$ e Longitude $50^{\circ} 48^{\prime} \mathrm{O}$ ), no ano de 2005 , e mantida em laboratório, segundo a metodologia descrita por Arioli et al. (2007). Os inseticidas e doses avaliados constam na Tabela 1.

Efeito de inseticidas sobre ovos de Grapholita molesta aplicados em pré-oviposição. Ponteiros de pessegueiro da cv. Chimarrita foram colhidos em pomar experimental, 30 dias após a última aplicação de inseticidas, e levados ao laboratório onde foram mergulhados por cinco segundos em 1L de calda contendo solução inseticida (Tabela 1). Após secagem à sombra por duas horas, os ponteiros foram transferidos para gaiolas confeccionadas com recipiente plástico de $300 \mathrm{~mL}$ ( $11 \mathrm{~cm}$ de altura $\times 5 \mathrm{~cm}$ de diâmetro) contendo $30 \mathrm{~mL}$ de ágar/água a 1,5\% para sustentar o ramo em posição vertical. Posteriormente, dois casais de G. molesta com três a cinco dias de idade foram confinados em cada gaiola, utilizando-se de um copo com capacidade de $120 \mathrm{~mL}(5 \mathrm{~cm}$ altura x $5 \mathrm{~cm}$ de diâmetro), em posição invertida para vedar a parte superior da gaiola. As gaiolas foram mantidas em sala de criação por $24 \mathrm{~h}$. Após este período, os ponteiros foram retirados das gaiolas, e as folhas com ovos foram cortadas e transferidas para placas de Petri $(1,3 \mathrm{~cm}$ de altura $\mathrm{x} 6 \mathrm{~cm}$ de diâmetro) contendo no interior da sua base papelfiltro umedecido. Posteriormente, as placas de Petri contendo os ovos foram acondicionas em câmara climatizada tipo BOD (temperatura de $25 \pm 1^{\circ} \mathrm{C}$, UR de $70 \pm 10 \%$ e fotofase de 16 horas). A eclosão das lagartas foi avaliada diariamente, por um período de cinco dias, utilizando-se de microscópio estereoscópico (10x de aumento).

Efeito de inseticidas sobre ovos de Grapholita molesta aplicados em pós-oviposição. Adultos de G. molesta com idade entre três e sete dias foram introduzidos em uma gaiola confeccionada com garrafa de polietileno (PET) de $2.000 \mathrm{~mL}$, onde foram mantidos por $24 \mathrm{~h}$. Passado este período, os adultos foram retirados, e as posturas, acondicionadas em câmara climatizada, onde permaneceram por $24 \mathrm{~h}$ para obtenção de ovos com 24 a $48 \mathrm{~h}$ de idade. Em seguida, as 
posturas foram recortadas, confeccionando-se 240 cartelas contendo 10 ovos cada, as quais foram mergulhadas por cinco segundos em 1L de solução inseticida contendo o respectivo tratamento. Após permanecerem duas horas à sombra para secagem, os ovos foram acondicionados em placas de Petri $(1,3 \mathrm{~cm}$ de altura x $6 \mathrm{~cm}$ de diâmetro) no interior de uma câmara climatizada, avaliando-se diariamente a eclosão das lagartas sob microscópio estereoscópico (aumento de 10x) durante cinco dias.

O delineamento experimental foi o inteiramente casualizado, em esquema fatorial com oito (tratamento) x dois (método de aplicação pré e pós-oviposição), empregando-se 30 repetições de 10 ovos cada, totalizando 300 ovos por forma de aplicação, para cada tratamento.

Efeito sobre lagartas de Grapholita molesta eclodidas dos ovos previamente tratados. As lagartas recém-eclodidas dos ovos previamente tratados com inseticidas que não apresentaram efeito ovicida, foram transferidas para tubos de vidro de fundo chato $(8,5 \mathrm{~cm}$ de comprimento $\times 2,5$ $\mathrm{cm}$ de diâmetro), contendo dieta artificial (ARIOLI et al., 2007) e tamponado com algodão hidrófugo. Avaliou-se a viabilidade da fase de lagarta e de pupa. O delineamento experimental foi inteiramente casualizado, empregando-se cinco repetições de 10 lagartas cada, totalizando 50 lagartas por tratamento.

Efeito de inseticidas sobre lagartas recémeclodidas de Grapholita molesta inoculadas em frutos e ponteiros de macieira e pessegueiro. Frutos e ponteiros de macieira cv. Gala e de pessegueiro cv. Eragil foram colhidos em pomar comercial, trinta dias após a última aplicação de inseticidas, sendo levados ao laboratório, onde foram mergulhados por cinco segundos em 1L de calda contendo solução inseticida. Após permanecerem por duas horas à sombra para a secagem, os ponteiros foram transferidos para recipientes de plástico com capacidade de 300 $\mathrm{mL}(11 \mathrm{~cm}$ de altura $\times 5 \mathrm{~cm}$ de diâmetro) contendo $30 \mathrm{~mL}$ de ágar/água a $1,5 \%$, e os frutos foram individualizados em recipientes de $500 \mathrm{~mL}$ (7 $\mathrm{cm}$ de altura $\mathrm{x} 8,5 \mathrm{~cm}$ de diâmetro), posicionados lateralmente sobre uma tampa de garrafa PET. Em cada estrutura vegetal, foi inoculada, com o auxílio de um pincel, uma lagarta recém-eclodida na região lateral dos frutos e no ápice dos ponteiros. Após a inoculação, os recipientes contendo os frutos e ponteiros foram fechados, respectivamente, com tecido tipo "voile" (18 x $18 \mathrm{~cm})$ e um copo com capacidade de $120 \mathrm{~mL}(5 \mathrm{~cm}$ altura x $5 \mathrm{~cm}$ de diâmetro), em posição invertida. A capacidade de penetração e a mortalidade das lagartas foram avaliadas aos 7 dias após a inoculação.

O delineamento experimental foi o inteiramente casualizado, em esquema fatorial: 8 (tratamento) x 4 (estrutura vegetal), empregando-se 5 repetições de 10 lagartas, totalizando 50 lagartas por estrutura vegetal (frutos e ponteiros de macieira e pessegueiro) para cada tratamento.

Efeito sobre adultos de Grapholita molesta. $\mathrm{O}$ efeito de inseticidas sobre adultos de $G$. molesta foi avaliado adaptando-se a metodologia descrita por Riedl et al. (1985). Adultos machos e fêmeas, com idade entre 0 e 72 horas, foram colados em cartões confeccionados com piso adesivo $(7 \mathrm{~cm}$ de comprimento x $3 \mathrm{~cm}$ de altura). Em seguida, os insetos foram pulverizados em torre de Potter calibrada para uma deposição média de $1,56 \mathrm{mg} . \mathrm{cm}^{-2}$ de resíduo úmido (pressão de $0,703 \mathrm{~kg} . \mathrm{cm}^{-2}$ e volume de calda de $2 \mathrm{~mL}$ ). Após a aplicação, os cartões foram colocados em bandejas e mantidos em sala de criação, avaliando-se a mortalidade a cada 24 horas, por um período de três dias. Para fins de padronização, nas avaliações, foram considerados mortos os insetos que não se movimentaram ao toque das cerdas de um pincel.

O delineamento experimental foi o inteiramente casualizado, utilizando-se de cinco repetições de 20 adultos, totalizando 100 adultos de cada sexo para cada tratamento.

Análise estatística. Para a análise estatística, os dados foram testados quanto à normalidade, utilizando o teste de Shapiro-Wilk (1965) e de homocedasticidade ou homogeneidade da variância dos erros, por Bartlett (1937) e Hartley (1950). Os dados que não apresentaram distribuição normal ou homogeneidade da variância (número de lagartas vivas) foram transformados em raiz de $\mathrm{X}+0,5$, e os dados expressos em porcentagem (mortalidade dos ovos), transformados em arcseno $\mathrm{x} / 100^{0,5}$. As médias dos tratamentos foram comparadas pelo teste de Tukey, ao nível de $5 \%$ de probabilidade de erro $(\mathrm{P}<0,05)$, utilizando o software Statistica 7.0 (STATSOFT, 2004). A porcentagem de controle dos inseticidas foi calculada pela fórmula de Abbott (1925). 


\section{RESULTADOS E DISCUSSÃO}

Efeito de inseticidas sobre ovos de $G$. molesta em pré-oviposição e pós-oviposição. Acetamiprido, fosmete, espinetoram e novalurom proporcionaram uma mortalidade dos ovos de $90,3 \%, 97,7 \%, 94,3 \%, 91,0 \%$ e $100,0 \%, 98,0 \%$, $96,3 \%$ e $86,0 \%$ quando aplicados em pré e pósoviposição, respectivamente (Tabela 2). Os resultados obtidos para o acetamiprido corroboram os observados por Brunner et al. (2005) e Arioli et al. (2007) para Cydia pomonella (Walsingham, 1897) e G. molesta, respectivamente.

O etofenproxi, quando aplicado diretamente sobre os ovos, reduziu a eclosão das lagartas em $99,0 \%$, comparado com 75,0\% quando aplicado antes da oviposição (Tabela 2). A maior redução da eclosão das lagartas observada quando aplicado diretamente sobre os ovos está associada ao efeito de contato quando aplicado sobre esta fase de desenvolvimento. Botton et al. (2009) observaram redução de apenas 30,0\% na eclosão de lagartas de Bonagota salubricola (Meyrick, 1937) (Lepidoptera: Tortricidae) tratados com etofenproxi.

$\mathrm{O}$ inibidor da síntese de quitina novalurom ocasionou mortalidade de $91,0 \%$ e $86,0 \%$ quando aplicado em pré e pós-oviposição, respectivamente, apresentando controle superior ao clorantraniliprole e ao piriproxifem aplicados em pré-oviposição, com $66,0 \%$ e $59,7 \%$ e em pós-oviposição com $69,7 \%$ e $70,0 \%$, respectivamente (Tabela 2 ).

Magalhães e Walgenbach (2011), ao avaliarem a $\mathrm{CL}_{50}$ dos inseticidas acetamiprido, clorantraniliprole, novalurom e piriproxifem sobre ovos de $G$. molesta, observaram que os produtos mais tóxicos foram acetamiprido e novalurom. $\mathrm{O}$ efeito ovicida dos inibidores da síntese de quitina novalurom e diflubenzurom também foi demonstrado, respectivamente, em Ostrinia nubilalis Hubner, 1796 (Lepidoptera: Crambidae) (BOITEAU; NORONHA, 2007) e Spodoptera littoralis (Boisduval, 1833) (Lepidoptera: Noctuidae) (ASSAL et al., 1983). Entretanto, Neto e Silva et al. (2011) observaram que o novalurom aplicado em pré e pós-oviposição reduziu em $23,0 \%$ e $28,0 \%$ a eclosão das lagartas de G. molesta, respectivamente.

Embora clorantraniliprole e piriproxifem tenham apresentado controle inferior aos demais tratamentos, ambos causaram mortalidade superior à testemunha. Quando foi avaliada a mortalidade das lagartas que eclodiram dos ovos tratados, tanto clorantraniliprole quanto piriproxifem não diferiram da testemunha, indicando que estas doses, quando aplicadas em pré e pós-oviposição, não apresentam efeito sobre as fases posteriores de desenvolvimento (Tabela 3).

Efeito de inseticidas sobre lagartas recémeclodidas de Grapholita molesta inoculadas em frutos e ponteiros de macieira e pessegueiro. Em ponteiros de macieira e pessegueiro, todos os inseticidas apresentaram controle das lagartas superior a $90 \%$, com exceção do piriproxifem, que causou uma mortalidade larval de $31 \%$ e $4 \%$, respectivamente, não diferindo da testemunha (Tabela 4). Magalhães e Walgenbach (2011), ao avaliarem a dose letal de inseticidas para lagartas recém-eclodidas de $G$. molesta, demonstraram que o regulador de crescimento piriproxifem foi pouco tóxico para a espécie, sendo que a maior dose testada $\left(4,8 \mathrm{~g} 100 \mathrm{~L}^{-1}\right)$ não foi suficiente para matar $50 \%$ dos indivíduos. Jones et al. (2010) também reportaram elevada toxicidade de acetamiprido, clorantraniliprole e espinetoram para lagartas de $G$. molesta.

Os inseticidas acetamiprido, clorantraniliprole, etofenproxi, fosmete e espinetoram aplicados em frutos de macieira causaram mortalidade de $100,0 \%$, $79,0 \%, 76,0 \%, 97,0 \%$ e $100,0 \%$, respectivamente, não havendo diferença significativa entre os produtos. O piriproxifem e o novalurom não diferiram da testemunha. Em frutos de pessegueiro, o inibidor da síntese de quitina novalurom, apesar de ocasionar mortalidade superior à testemunha, apresentou controle inferior aos demais tratamentos (Tabela 4).

O controle obtido com o organofosforado fosmete (padrão no controle da G. molesta) assemelhou-se aos observados por Arioli et al. (2004, 2007), que observaram $80,0 \%$ e $90,0 \%$ de controle das lagartas que foram inoculadas em frutos de pessegueiro e macieira, respectivamente.

De modo semelhante ao observado neste trabalho, Arioli et al. (2007) demonstraram que acetamiprido foi eficiente no controle de lagartas de $G$. molesta em macieira. Os autores verificaram que o produto ocasionou $97,0 \%$ de mortalidade em condições de laboratório e $88,0 \%$ de controle em pomar comercial.

Ao contrário do que ocorreu sobre os ovos (Tabela 2), o inseticida clorantraniliprole, quando aplicado sobre os frutos, foi superior no controle das lagartas quando comparado ao novalurom. Estes resultados corroboram os observados por Magalhães e Walgenbach (2011), que demonstraram que clorantraniliprole é mais tóxico a lagartas de $G$. molesta, quando comparado ao novalurom.

$\mathrm{O}$ fato de o inibidor da síntese de quitina novalurom causar maior mortalidade das lagartas 
que foram inoculadas nos ponteiros do que nos frutos tratados pode ser explicado pelo comportamento alimentar da espécie. As lagartas, ao penetrarem nos ponteiros de macieira ( $5 \mathrm{~h} 23$ ) ficam maior tempo expostas e, consequentemente, ingerem maior quantidade de produto do que em frutos ( $3 \mathrm{~h}$ 7) (CHAVES, 2013). Siqueira e Grutzmacher (2005) obtiveram alta eficiência de controle de $G$. molesta em ponteiros de pessegueiro após pulverização do inseticida inibidor da síntese de quitina lufenurom. No entanto, em frutos de macieira tratados com novalurom e lufenurom, a mortalidade foi inferior a 60\% (NETO; SILVA et al., 2011). Dessa forma, o substrato onde o inseticida é aplicado (ponteiros e frutos de macieira e pessegueiro) interfere na eficiência do produto devido ao hábito alimentar do inseto-praga. Além disso, lagartas recém-eclodidas de $C$. pomonella não ingerem a casca de frutos de macieira, alimentando-se somente após atingir o interior dos frutos (GRANGER, 2011). Devido às semelhanças entre estas espécies, em hipótese, $G$. molesta também exibe esse comportamento de não ingerir a casca do fruto.

Nas estruturas vegetais tratadas com acetamiprido, clorantraniliprole, etofenproxi, fosmete e espinetoram, houve redução significativa no número de lagartas que penetraram nos frutos (Tabela 5). Em contrapartida, nos frutos de macieira e pessegueiro tratados com novalurom, houve penetração de $60,0 \%$ e $68,0 \%$ das lagartas, respectivamente. $\mathrm{O}$ inseticida piriproxifem não diferiu significativamente da testemunha, havendo penetração superior a 76,0\% (Tabela 5).

O fato de os inseticidas reguladores de crescimento não evitarem que ocorresse a penetração, deve-se ao seu modo de ação. Após a ingestão, para que ocorra a morte do inseto, é necessário que os mesmos troquem de instar. G. molesta apresenta de quatro a cinco instares, dependendo da temperatura e fonte de alimento em que se desenvolve (RUSSEL; BOUZOUANE, 1989), sendo que cada instar dura entre dois e três dias (PETERSON; HAEUSSLER, 1930). Neste sentido, o emprego do novalurom seria mais indicado quando o objetivo é proteger os ponteiros (início do ciclo de desenvolvimento da cultura). Quando há a necessidade de proteger os frutos, devem-se priorizar inseticidas com ação de contato que evitem a penetração das lagartas nestas estruturas (Tabela 5).

Efeito de inseticidas sobre adultos de G. molesta. Os inseticidas etofenproxi e fosmete foram os únicos tóxicos, tanto para as fêmeas quanto para os machos adultos de G. molesta. O espinetoram apresentou efeito somente sobre os machos (Tabela 6). Semelhantemente ao observado neste trabalho, Santos et al. (2011) obtiveram 75,0\% de mortalidade dos adultos de G. molesta 72 horas após a pulverização de fosmete em gaiolas contendo adultos fixados em plantas de macieira.

Os inseticidas etofenproxi, fosmete e espinetoram foram menos tóxicos para as fêmeas de G. molesta, quando comparado aos machos (Tabela 6). Estes resultados corroboram os observados por Riedl et al. (1985) e Knight e Hull (1989), que verificaram que machos de C. pomonella (Lepidoptera: Tortricidae) e Platynota idaeusalis (Walker, 1859) (Lepidoptera: Tortricidae) são mais suscetíveis à aplicação de inseticidas quando comparado às fêmeas. Segundo Knight e Hull (1989), a maior tolerância das fêmeas está relacionada a diferenças morfológicas, como quantidade de escamas que podem reduzir a penetração dos produtos e as diferenças bioquímicas que podem permitir que as fêmeas se desintoxiquem mais facilmente.

$\mathrm{Na}$ avaliação realizada 24 horas após a pulverização (HAP), nenhum dos tratamentos causou mortalidade significativa. Etofenproxi causou 40,0\% de mortalidade das fêmeas 48 HAP, sendo semelhante ao registrado pelo fosmete $72 \mathrm{HAP}$ (39\%). A maior mortalidade foi observada 72HAP com o inseticida etofenproxi (59,0\%) (Tabela 6). Nos machos, apenas etofenproxi causou mortalidade superior à testemunha 24HAP. Entretanto, 48HAP o número de adultos mortos após a pulverização com fosmete e etofenproxi não diferiu significativamente. A mortalidade de adultos registrada pelo inseticida espinetoram em fêmeas foi equivalente ao fosmete e ao etofenproxi somente após 72HAP.

Magalhães e Walgenbach (2011) verificaram que, para adultos machos de C. pomonella e G. molesta, a $\mathrm{CL}_{50}$ de acetamiprido foi de $4 \mathrm{~g}$ i.a $100 \mathrm{~L}^{-1}$ e $6 \mathrm{~g}$ i.a $100 \mathrm{~L}^{-1}$ de água, respectivamente. Os mesmos autores verificaram que acetamiprido foi mais tóxico para $C$. pomonella que o organofosforado azinfós metil. Já para $G$. molesta, a toxicidade desses dois inseticidas não diferiu. Entretanto, Brunner et al. (2005), ao avaliarem a $\mathrm{CL}_{50}$ de inseticidas neonicotinoides (acetamiprido, clotianidina e tiacloprid) para adultos de $C$. pomonella, demonstraram que os três inseticidas apresentaram baixa toxicidade em relação ao organofosforado azinfós metil. Neste trabalho, o máximo controle obtido com acetamiprido sobre adultos machos de G. molesta foi de 17,0\%, utilizando-se de $8 \mathrm{~g}$ i.a $100 \mathrm{~L}^{-1}$ de água (Tabela 6).

Com base nos resultados deste trabalho, os inseticidas etofenproxi, fosmete e espinetoram apresentam efeito significativo sobre ovos, lagartas 
e adultos de G. molesta, enquanto o acetamiprido, apesar de não ter apresentado efeito em adultos, é uma alternativa para o controle de ovos e lagartas.

Devido ao modo de ação dos inseticidas, o novalurom deve ser direcionado às fases de ovo $\mathrm{e}$ lagartas no início do desenvolvimento das culturas, quando ainda não há presença de frutos. Por outro lado, o clorantraniliprole pode ser direcionado para o controle da fase de lagarta durante todo o ciclo da cultura. Por também apresentarem efeitos sobre os adultos, os inseticidas fosmete, etofenproxi e espinetoram podem ser alternativas em tratamentos visando ao controle de adultos antes da aplicação de feromônios sexuais, visando a reduzir a população de fêmeas fecundadas na área tratada (PASTORI et al., 2012).

TABELA 1 - Produto comercial, ingrediente ativo, dose, grupo químico e classe toxicológica dos inseticidas utilizados no experimento visando ao controle de Grapholita molesta em laboratório (T: $25 \pm 1^{\circ} \mathrm{C}$, UR de $70 \pm 10 \%$ e fotofase de 16 horas).

\begin{tabular}{cccccc}
\hline \multirow{2}{*}{ Ingrediente ativo } & \multirow{2}{*}{ Produto comercial $^{2}$} & \multicolumn{2}{c}{ Dose $^{1}$} & \multirow{2}{*}{ Grupo químico } & \multirow{2}{*}{ Classe toxicológica } \\
\cline { 3 - 4 } & & i.a. & p.c. & & \\
\hline Acetamiprido & Mospilan $^{\circledR}$ & 8 & 40 & Neonicotinoide & III \\
\hline Clorantraniliprole & Altacor $^{\circledR}$ & 4,9 & 14 & Antranilamida & III \\
\hline Etofenproxi & Trebon $^{\circledR} 100 \mathrm{SC}$ & 15 & 150 & Éter difenílico & III \\
\hline Fosmete & Imidan $^{\circledR} 500 \mathrm{WP}$ & 100 & 200 & Organofosforado & I \\
\hline Novalurom & $\operatorname{Rimon}^{\circledR} 100 \mathrm{EC}$ & 4,0 & 40 & Benzoilureia & IV \\
\hline Piriproxifem & $\operatorname{Tiger}^{\circledR} 100 \mathrm{EC}$ & 10 & 100 & Éter piridiloxipropílico & I \\
\hline Spinetoram & Delegate $^{\circledR}$ WG & 3,75 & 15 & Espinosinas & III \\
\hline
\end{tabular}

'gramas de ingrediente ativo (i.a.) ou $\mathrm{mL}$ de produto comercial (p.c.) por 100 litros de água.

TABELA2 - Efeito de inseticidas sobre ovos de Grapholita molesta quando aplicados em pré e pós-oviposição em laboratório (T: $25 \pm 1^{\circ} \mathrm{C}$, UR de $70 \pm 10 \%$ e fotofase de 16 horas).

\begin{tabular}{cccccc}
\hline \multirow{2}{*}{ Tratamento } & Concentração $^{1}$ & \multicolumn{2}{c}{ Pré-oviposição } & \multicolumn{2}{c}{ Pós-oviposição } \\
\cline { 2 - 6 } & i.a. & Mortalidade (\%) & $\% \mathrm{C}^{2}$ & Mortalidade (\%) & $\% \mathrm{C}$ \\
\hline Acetamiprido & 8 & $90,3 \pm 2,46 \mathrm{Aab}$ & 90,0 & $100,0 \pm 0.00 \mathrm{Aa}$ & 100,0 \\
\hline Clorantraniliprole & 4,9 & $66,0 \pm 5,08 \mathrm{Ac}$ & 62,3 & $69,7 \pm 5,06 \mathrm{Ac}$ & 61,0 \\
\hline Espinetoram & 3,75 & $94,3 \pm 1,49 \mathrm{Aa}$ & 94,1 & $96,3 \pm 1,15 \mathrm{Aab}$ & 96,0 \\
\hline Etofenproxi & 15 & $80,0 \pm 2,83 \mathrm{Bbc}$ & 75,1 & $99,3 \pm 0,46 \mathrm{Aab}$ & 99,3 \\
\hline Fosmete & 100 & $97,7 \pm 1,03 \mathrm{Aa}$ & 97,6 & $98,0 \pm 1,38 \mathrm{Aab}$ & 97,8 \\
\hline Novalurom & 4,0 & $91,0 \pm 2,85 \mathrm{Aa}$ & 90,7 & $86,0 \pm 3,23 \mathrm{Ab}$ & 84,7 \\
\hline Piriproxifem & 10 & $59,7 \pm 5,39 \mathrm{Ac}$ & 58,1 & $70,0 \pm 4,54 \mathrm{Ac}$ & 67,2 \\
\hline Testemunha & - & $3,7 \pm 1,76 \mathrm{Ad}$ & - & $8,7 \pm 2,07 \mathrm{Ad}$ & - \\
\hline
\end{tabular}

${ }^{1}$ gramas de ingrediente ativo (i.a.) por 100 litros de água. ${ }^{2} \% \mathrm{C}$ - Mortalidade corrigida por Abbott (1925).Médias ( \pm EP) seguidas da mesma letra, minúscula na coluna (inseticidas) e maiúscula na linha (forma aplicação), não diferem entre si, pelo teste de Tukey (p<0,05).

TABELA 3 - Viabilidade de lagartas e pupas de Grapholita molesta provenientes de ovos tratados com inseticidas em pré e pós-oviposição em laboratório (T: $25 \pm 1^{\circ} \mathrm{C}$, UR de $70 \pm 10 \%$ e fotofase de 16 horas).

\begin{tabular}{|c|c|c|c|c|c|}
\hline \multirow[b]{3}{*}{ Tratamento } & \multirow{3}{*}{$\begin{array}{c}\text { Concentração }^{1} \\
\text { i.a. }\end{array}$} & \multicolumn{4}{|c|}{ Viabilidade (\%) } \\
\hline & & \multicolumn{2}{|c|}{ Lagartas } & \multicolumn{2}{|c|}{ Pupas } \\
\hline & & $\begin{array}{c}\text { Aplicação } \\
\text { Pré-oviposição }\end{array}$ & $\begin{array}{c}\text { Aplicação } \\
\text { Pós-oviposicão }\end{array}$ & $\begin{array}{c}\text { Aplicação } \\
\text { Pré-oviposição }\end{array}$ & $\begin{array}{c}\text { Aplicação } \\
\text { Pós-oviposicão }\end{array}$ \\
\hline Clorantraniliprole & 4,9 & $64,0 \pm 5,1 \mathrm{Aa}^{2}$ & $76,0 \pm 4,00 \mathrm{Aa}$ & $54,0 \pm 12,08 \mathrm{Aa}$ & $72,0 \pm 6,63 \mathrm{Aa}$ \\
\hline Piriproxifem & 10 & $64,0 \pm 6,8 \mathrm{Aa}$ & $84,0 \pm 6,78 \mathrm{Aa}$ & $50,0 \pm 7,07 \mathrm{Aa}$ & $70,0 \pm 7,74 \mathrm{Aa}$ \\
\hline Testemunha & - & $82,0 \pm 11,13 \mathrm{Aa}$ & $80,0 \pm 8,36 \mathrm{Aa}$ & $76,0 \pm 9,79 \mathrm{Aa}$ & $74,0 \pm 8,71 \mathrm{Aa}$ \\
\hline
\end{tabular}


象 웅

焉

究

की

జ్

잉

党

\& 0

तै 占

: ๘ี

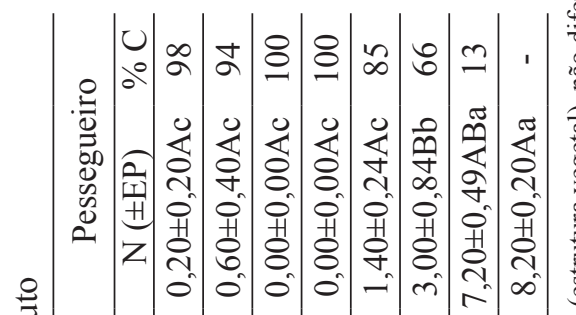

ชิ

율

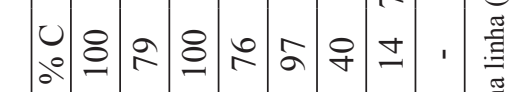

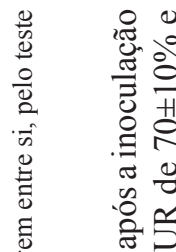

$\frac{10}{\circ}$

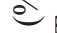

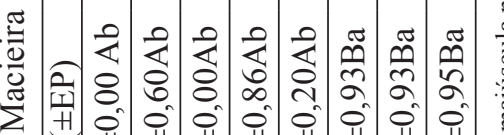

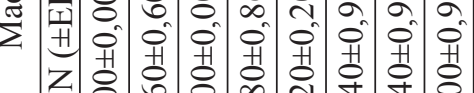

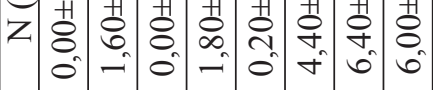

응

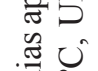

둥

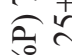

อั

赵.

递

릉

플

䍃

要

(1)

苋.

긍

ฮี

0.

छี

䒿

旁 హ్d

$\stackrel{2}{2}$

बิ

ฮ

Uి

离

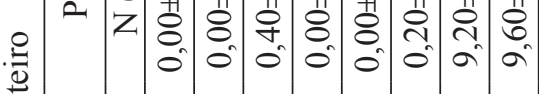

:

ㅇำ

율

莺

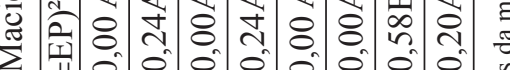

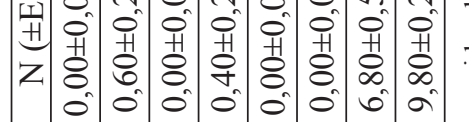

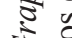

蒂

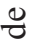

迅

응

章

$\stackrel{\square}{*}$

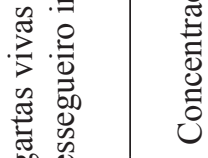

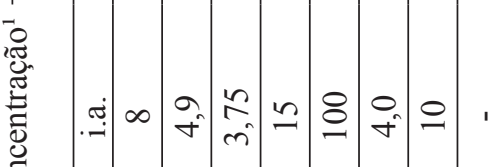

\%

这

震

गु.

茾芯 吾

$\sum^{\infty}$

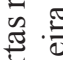

త్ర్ల

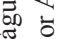

西

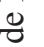

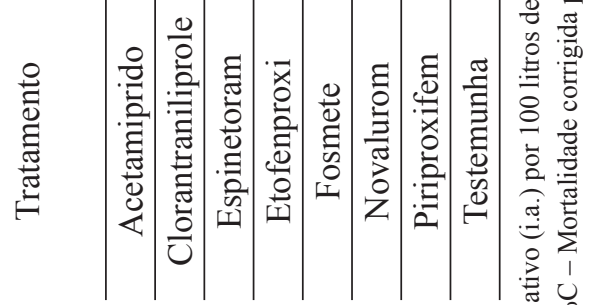

$\approx \%$

商总

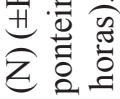

은

焉

造造

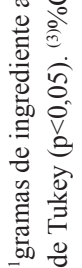

章

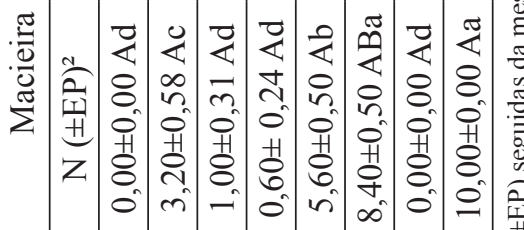

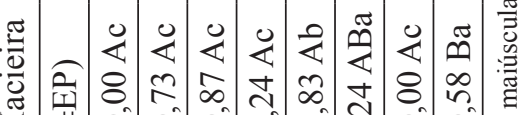

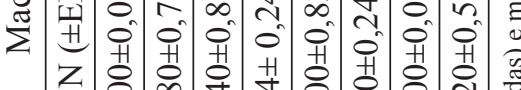

ar + 0 +

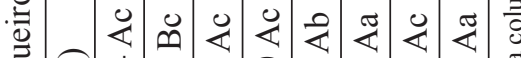

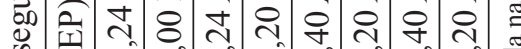

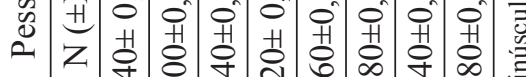

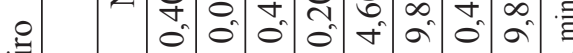

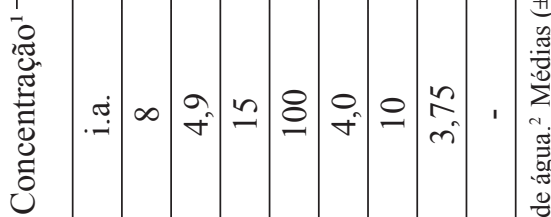

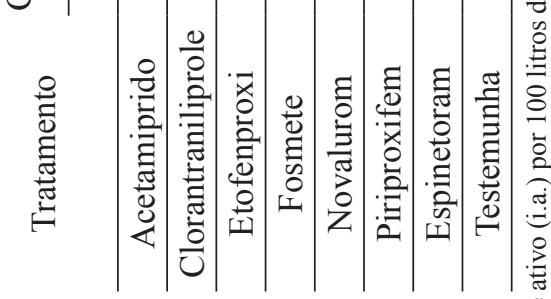

要要

新

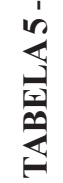

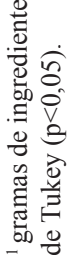

Rev. Bras. Frutic., Jaboticabal - SP, v. 36, n. 4, p. 842-852, Dezembro 2014 


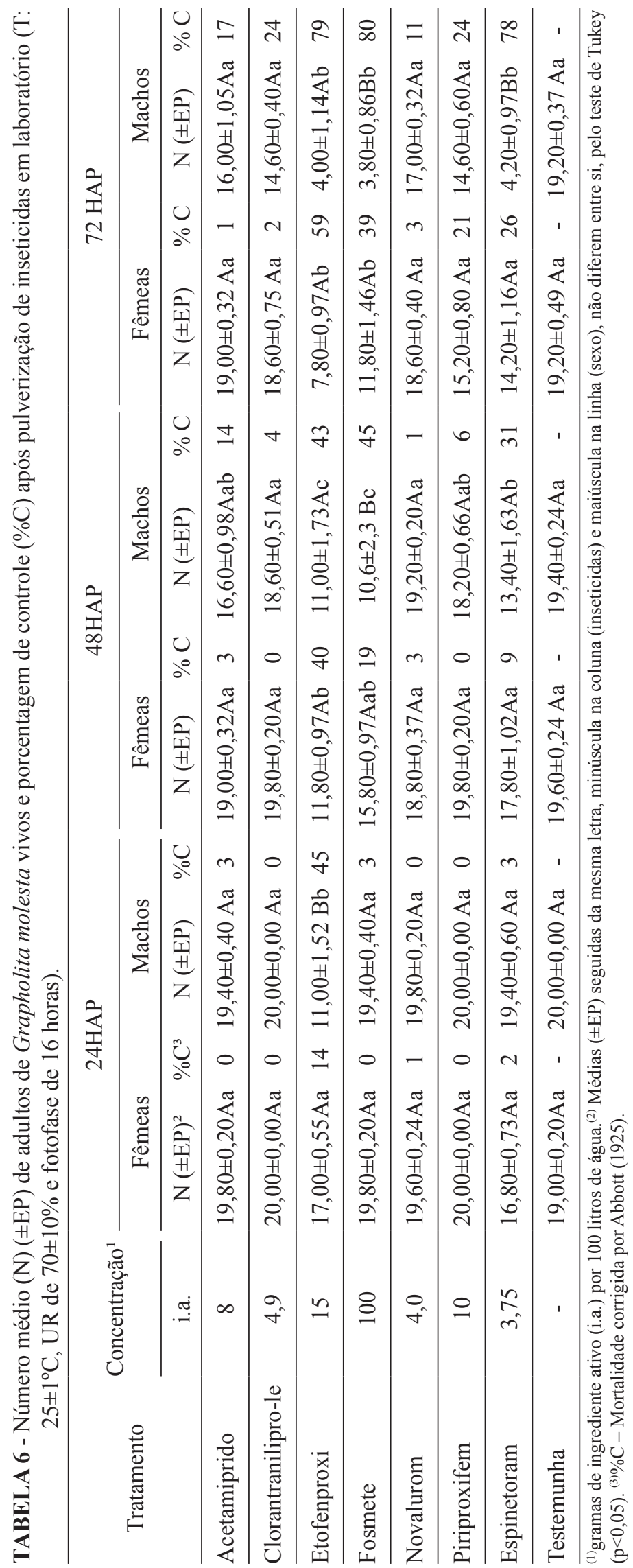




\section{CONCLUSÕES}

Os inseticidas Acetamiprido $\left(8 \mathrm{~g} 100 \mathrm{~L}^{-1}\right)$,

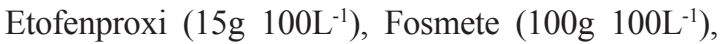
Novalurom (4,0g 100 $\left.\mathrm{L}^{-1}\right)$ e Espinetoram (3,75g $100 \mathrm{~L}^{-1}$ ) afetam ovos de Grapholita molesta quando aplicados em pré ou pós-oviposição.

Acetamiprido $\left(8 \mathrm{~g} 100 \mathrm{~L}^{-1}\right)$, Clorantraniliprole

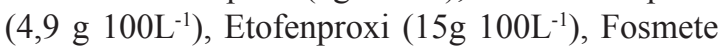
$\left(100 \mathrm{~g} 100 \mathrm{~L}^{-1}\right)$ e Espinetoram $\left(3,75 \mathrm{~g} 100 \mathrm{~L}^{-1}\right)$ aplicados sobre ponteiros e frutos de macieira e pessegueiro, em laboratório, são eficazes no controle de lagartas de G. molesta, reduzindo a penetração do inseto nas estruturas vegetais.

O inseticida Novalurom (4,0g $\left.100 \mathrm{~L}^{-1}\right)$ aplicado em ponteiros causa maior mortalidade de lagartas de $G$. molesta quando comparado aos frutos de macieira e pessegueiro.

Etofenproxi (15 g 100L $\left.\mathrm{L}^{-1}\right)$, Fosmete $(100 \mathrm{~g}$ $\left.100 \mathrm{~L}^{-1}\right)$ e Espinetoram $\left(3,75 \mathrm{~g} 100 \mathrm{~L}^{-1}\right)$ são letais aos adultos machos de $G$. molesta.

\section{REFERÊNCIAS}

ABBOTT, W. S. A. Method of computing the effectiveness of an insecticide. Journal of Economic Entomology, College Park, v.18, n.1, p.265-267, 1925.

AGNELlO, A. M.; ATANASSOV, A.; BERGH, J. C.; BIDDINGER, D. J.; GUT, L. J.; HAAS, M. J.; HARPER, J. K.; HOGMIRE, H. W.; HULL, L. A.; KIME, L. F.; KRAWCZYK, G.; MCGHEE, P. S.; NYROP, J. P.; REISSIG, W. H.; SHEARER, P. W.; STRAUB, R. W.; VILLANUEVA, R. T.; WALGENBACH, J. F. Reduced-Risk Pest Management Programs for Eastern U.S. Apple and Peach Orchards: A 4-Year Regional Project. American Entomologist, Annapolis, v. 55, n. 3, p. 184-197, 2009.

AGROFIT. Sistemas de agrotóxicos fitossanitários. Brasília: Ministério da Agricultura, Pecuária e Abastecimento. Disponível em: <http://extranet. agricultura.gov.br/agrofit_cons/principal_agrofit cons $>$ Acesso em: 21 jan. 2013.

ANVISA. Reavaliações de agrotóxicos 2010: Fosmete. Disponível em: <http://s.anvisa.gov.br/ wps/s/r/cjL >. Acesso em: 08 jan. 2012.
ARIOLI, C. J.; BOTTON, M.; CARVALHO, G. A. Controle químico da Grapholita molesta (Busck) (Lepidoptera: Tortricidae) na cultura do pessegueiro. Ciência Rural, Santa Maria, v. 34, n. 6, p. 1695 1700, 2004.

ARIOLI, J. C.; MOLINARI, F.; BOTTON, M.; GARCIA, M. S. Técnica de criação de Grapholita molesta (Busck, 1916) (Lepidoptera: Tortricidae) em laboratório utilizando dieta artificial para produção de insetos visando a estudos de comportamento e controle. Bento Gonçalves: Embrapa Uva e Vinho, 2007. 14 p. (Boletim de pesquisa e desenvolvimento, 13).

ASSAL, O. M.; RADWAN, H. S. A.; SAMY, M. E. Egg hatch inhibition in the Cotton Leafworm with certain IGRS and synthetic pyrethroids. Journal of Applied Entomology, Hamburg, v. 95, p. 259-263, 1983.

BARTLETT, M. S. Properties of sufficiency and statistical tests. Proceedings of the Royal Society A: Mathematical, Physical \& Engineering Sciences, London, v. 160, p. 268-282, 1937.

BASSI, A.; RISON, J. L.; WILES, J. A. Chlorantraniliprole (DPX-E2Y45, Rynaxypyr®, Coragen $\left({ }^{\circledR}\right)$, a new diamide insecticide for control of codling moth (Cydia pomonella), Colorado potato (Leptinotarsa decemlineata) and European grapevine moth (lobesia botrana). In: SLOVENIAN CONFERENCE ON PLANT PROTECTION, 9., 2009, Nova Gorica. v.1, p.39-45.

BOITEAU, G.; NORONHA, C. Topical, residual and ovicidal contact toxicity of three reduced-risk inseticides against the European corn borer, Ostrinia nubilalis (Lepidoptera: Crambidae), on potato. Pest Management Science, West Sussex, v. 63, p.12301238, 2007.

BOTTON, M.; ARIOLI, C. J.; RINGENBERG, R.; MORANDI-FILHO, W. J. Controle químico de Bonagota salubricola (Meyrick, 1937) (Lepidoptera: Tortricidae) em laboratório e pomar de macieira. Arquivos do Instituto Biológico, São Paulo, v. 76, n. 2, p. 225-231, 2009. 
BOTTON, M.; NAVA, D. E.; ARIOLI, C. J.; GRUTZMACHER, A. D.; GARCIA, M. S. Bioecologia, monitoramento e controle da mariposa-oriental na cultura do pessegueiro no Rio Grande do Sul. Bento Gonçalves: Embrapa Uva e Vinho, 2011. 11p. (Circular Técnica, 86).

BRUNNER, J. F.; BEERS, E. H.; DUNLEY, J. E.; DOERR, M.; GRANGER, K. Role of neonicotinyl insecticides in Washington apple integrated pest management. Part I. Controlo f lepidopteran pests. Journal of Insect Science, Washington, v.5, n.14, p.1-10, 2005.

CHAVES, C. C. Local de oviposição, tempo de penetração, efeito de inseticidas e parasitoides larvais associados à Grapholita molesta (Busck, 1916) (Lepidoptera: Tortricidae) em macieira e pessegueiro. 2013. 76 f. Dissertação (Mestrado em Fitossanidade) - Faculdade de Agronomia Eliseu Maciel, Universidade Federal de Pelotas, Pelotas, 2013.

GRANGER, K. Integrating new insecticides. Washington: Washington State University, 2011. Disponível em: $<$ http://pmtp.wsu.edu/INI presentation.html $>$ Acesso em: 10 nov. 2012.

GUERRA, D. S.; MARODIN, G. A. B.; ZANINI, C. L. D.; ARGENTA, F.; GRASELLI, V.; NUNES, J. L. S. Utilização de pesticidas na produção integrada de pêssegos 'Marli', nos sistemas de produção integrada e convencional. Revista Brasileira de Fruticultura, Jaboticabal, v. 29, n.1, p. 91-95, 2007.

HARTLEY, H. O. The use of range in analysis of variance. Biometrika, London, v. 37, p.271-280, 1950.

JONES, M. M.; ROBERTSON, J. L.; RICHARD, A.; WEINZIERL, R. A. Susceptibility of Oriental Fruit Moth (Lepidoptera: Tortricidae) larvae to selected reduced-risk insecticides. Journal of Economic Entomology, College Park, v. 103, n. 5, p.18151820, 2010.

KANGA, L. H. B.; PREE, D. J.; VAN LIER, J. L.; WALKER, G. M. Monitoring for resistance to organophosphorus, carbamate, and pyrethroid insecticides in the oriental fruit moth (Lepidoptera: Tortricidae). Canadian Entomologist, Ontario, v. 131, n. 4, p. 441-450, 1999.
KNIGHT, A. L.; HULL, L. A. Use of ser pheromone traps to monitor azinphosmethyl resistance in tufted apple bud moth (Lepidoptera: Tortricidae). Journal of Economic Entomology, College Park, v. 82, n. 4, p.1019-1026, 1989.

MAGALHÃES, L. C.; WALGENBACH, J. F. Life stage toxicity and residual activity of insecticides to codling moth and oriental fruit moth (Lepidoptera: Tortricidae). Journal of Economic Entomology, College Park, v. 104, n. 6, p.1950-1959, 2011.

MAPA. Ministério da Agricultura, Pecuária e Abastecimento. Instrução Normativa $\mathrm{n}^{\circ} 1$, de 4 de janeiro de 2013. Dispõe sobre o plano nacional de controle de resíduos e contaminantes em produtos de origem vegetal no ano-safra 2011/2012. Diário Oficial da União, Brasília- DF, v. 1, n. 4, p. 10, jan. 2013.

MONTEIRO, L. B.; HiCKeL, E. Pragas de importância econômica em fruteiras de caroço. In: MONTEIRO, L. B.; MAY de MIO, L. L.; SERRAT, B. M.; MOTTA, A. C. V.; CUQUEL, F.L. (Org.). Fruteiras de caroço: uma visão ecológica. Curitiba: Universidade Federal do Paraná, 2004. p. 223-264.

NETO-SILVA, O. A. B.; BOTTON, M.; GARCIA, M. S.; DA SILVA, A. Efeito de inseticidas reguladores de crescimento sobre ovos, lagartas e adultos de Grapholita molesta (Busck) (Lep.: Tortricidae). Revista Brasileira de Fruticultura, Jaboticabal, v. 33, n. 2, p. 420-428, 2011.

NORA, I.; HICKEL, E. D. Pragas da macieira. In: EPAGRI. A cultura da macieira. Florianópolis: Epagri, 2006. p. 463-521.

NUNES, J. L. S.; MARODIN, G. A. B. Inseticidas metoxifenozide e etofenproxi para o controle de mariposa-oriental (Grapholita molesta Busck, 1916) em produção integrada de pêssego. Revista Ceres, Viçosa, MG,v.54, n.316, p.511-516, 2007.

PASTORI, L. P.; ARIOLI, C. J.; BOTTON, M.; MONTEIRO, L. B.; STOLTMAN, L.; MAFRANETO, A. Integrated controlo $\mathrm{f}$ two tortricid (Lepidoptera) pests in apple orcrards with sex pheromones and insecticides. Revista Colombiana de Entomologia, Bogotá, v.38, n.2, p.224-230, 2012. 
PETERSON, A.; HAEUSSLER, G. J. Life history of the oriental peach moth at Riverton, N. J., in relation to temperature. Washington: United States Department of Agriculture, 1930. 38p. (Technical Bulletin, 183).

PREE, D. J.; WHITTY, K. J.; VAN DRIEL, L.; WALKER, G. M.; VAN DRIEL, L. Resistance to insecticides in oriental fruit moth populations (Grapholita molesta) from the Niagara Peninsula of Ontario. Canadian Entomologist, Ottawa, v.130, n.3, p.245-256, 1998.

RIEDL, H.; SEAMAN, A.; HENRIE, F. Monitoring susceptibility to azinphosmethyl in fiels populations of the Codling Moth (Lepidoptera: Tortricidae) with pheromone traps. Journal of Economic Entomology, College Park, v.78, n. 3, 692-699, 1985.

RUSSEL, D. A.; BOUZOUANE, R. The effect of diet, temperature and diapause on the number and identification of larval instars in the oriental fruit moth Grapholita molesta (Busck) (Lepidoptera: Tortricidae). Agronomie, Paris, v.9, p.919-926, 1989.

SALLES, L. A. B. A Mariposa-oriental, Grapholita molesta (Lepidoptera: Tortricidae). In: VILELA, E. F.; ZUCCHI, R. A.; CANTOR, F. (Ed.). Histórico e impacto das pragas introduzidas no Brasil. Ribeirão Preto: Holos, 2001. p.42-45.

SANTOS, R. S. S.; LEOLATO, L. S.; GIUSTINA, P. G. D.; SOUZA, R. B. Avaliação da mortalidade de adultos de Grapholita molesta sob condições de controle químico no campo. AGAPOMI, Vacaria, v. 206, p. 6-7, 2011.

SHAPIRO, S. S.; WILK, M. B. An analysis of variance test for normality (complete samples). Biometrika, London, v.52, n.3-4, p.591-611, 1965.

SHEARER, P. W.; USMANI, K. A. Sex-related response to organophosphorus and carbamate insecticides in adult oriental fruit moth, Grapholita molesta. Pest management Science, West Sussex, v. 57, n. 9, p. 822-826, 2001.
SIEGWART, M.; MONTEIRO, L. B.; MAUGIN, S.; OLIVARES, J.; CARVALHO, S. M.; SAUPHANOR, $B$. Tools for resistance monitoring in oriental fruit moth (Lepidoptera: Tortricidae) and first assessment in Brazilian populations. Journal of Economic Entomology, College Park, v. 104, n. 2, p. 636-645, 2011.

SIQUEIRA, P. R. E.; GRUTZMACHER, A. D. Avaliação de inseticidas para o controle da Grapholita molesta (Busck, 1916) (Tortricidae) em pomares de pessegueiro sob produção integrada na região da campanha do RS. Revista Brasileira de Agrociência, Pelotas, v.11, n.2, p. 185-191, 2005.

SPARKS, T. C.; CROUSE, G. D.; DURST, G. Natural products as inseticides: the biology, biochemistry and quantitative structure-activity relationships of spinosyns and spinosoids. Pest Management Science, West Sussex, v. 57, p. 896-905, 2001.

STATSOFT. Statistica: data analysis software system. Versão 7.0. Tulsa, 2004.

USMANI, K. A.; SHEARER, P. W. Susceptibility of male Oriental fruit moth (Lepidoptrea: Tortricidae) populations from New Jersey apple orchards to Azinophosmethyl. Journal of Economic Entomology, College Park, v.94, n.2, p.233-239, 2001.

YOKOYAMA, V.; MILLER, G. T. Potencial of pyriproxyfen as a quarantine treatment for codling moth and oriental fruit moth. Journal of Economic Entomology, College Park, v.84, n.3, p.942-947, 1991. 